\title{
PHOTOCHEMICAL GENERATION OF REDUCED QUINONE CATALYSTS OF PHOTOSYSTEM I CYCLIC PHOTOPHOSPHORYLATION ACTIVITY
}

\author{
Howard H. Robinson and Charles F. Yocum \\ Department of Cellular and Molecular Biology, Division of Biological Sciences, \\ The University of Michigan, Ann Arbor, MI 48109, U.S.A.
}

(Received 5 January 1978; accepted 17 March 1978)

\begin{abstract}
When reaction mixtures containing 9,10-anthraquinone-2-sulfonate and chloroplasts poisoned with 3-(3,4-dichlorophenyl)-1,1'-dimethylurea were illuminated with white light, photosystem I-catalyzed cyclic photophosphorylation was observed. Illumination of identical reaction mixtures with red light produced no ATP synthesis. This phenomenon is due to photoreduction of the anthraquinone which is supported by the electron donor activity of Tricine buffer. The photoreduction reaction was used to generate reduced catalysts (anthraquinone sulfonate, menadione bisulfite) of photosystem I cyclic photophosphorylation activity. The rates of ATP synthesis obtained by this method $(250-300 \mu \mathrm{mol} / \mathrm{h} \cdot \mathrm{mg}$ chlorophyll) indicate that sulfonated quinones are efficient mediators of cyclic electron transport around photosystem I. Although the activity catalyzed by these compounds is highly sensitive to dibromothymoquinone, very little decrease in activity is observed with antimycin $A$.
\end{abstract}

\section{INTRODUCTION}

Catalysts of photosystem I-dependent cyclic photophosphorylation reactions may be divided into two groups. The first group of catalysts is comprised of lipophilic proton and electron donors such as diaminodurene and phenazinemethosulfate. The second group of catalysts includes both electron donors (ferredoxin) and proton and electron donors such as menadione and 9,10-anthraquinone-2-fulfonate. These two groups of catalysts differ both with respect to the rates of ATP synthesis they produce, and with regard to their sensitivity to inhibition by the plastoquinone antagonist DBMIB*. The PS I cyclic reactions catalyzed by diaminodurene or phenazinemethosulfate can produce rates of ATP synthesis in excess of $1000 \mu \mathrm{mol} / \mathrm{h} \cdot \mathrm{mg}$ Chl (Nelson et al., 1970; Ouitrakul and Izawa, 1973), and addition of DBMIB to these reaction systems produces little, if any, inhibition of activity (Böhme et al., 1971). The rates of ATP synthesis obtained with ferredoxin and quinones are invariably lower $(20-200 \mu \mathrm{mol} / \mathrm{h} \cdot \mathrm{mg} \mathrm{Chl}$; Tagawa et al.. 1963; Voegeli et al., 1977), and the activity supported by these catalysts is sensitive to DBMIB. This result has been interpreted as indicating that native components of the thylakoid membrane, rather than the catalysts themselves, are involved in coupling of electron transport to photophosphorylation (Hauska et al., 1974).

Assay of the PS I cyclic photophosphorylation

*Abbreviations used are: DBMIB-dibromothymoquinone (2,5-dibromo-3-methyl-6-isopropyl-p-benzoquinone); PS I-photosystem I; DCMU-3-(3,4-dichlorophenyl)1,1'-dimethylurea; Chl-chlorophyll; AQS-9,10-anthraquinone-2-sulfonate. reactions catalyzed by ferredoxin and low-potential quinones is complicated. To prevent autooxidation of the added catalyst, the reaction must be carried out under anaerobic conditions, and the catalyst must be partially reduced to provide a source of reducing power to initiate the cycle. A number of investigators (Böhme et al., 1971; Hauska et al., 1974; Voegeli et al., 1977) have reported assay conditions for these reactions which involve purging the reaction mixtures for prolonged periods with inert gas. To permit photosynthetic reduction of the catalyst, the gassed mixtures are next illuminated. Finally, a second illumination period is used to drive the cyclic photophosphorylation reaction. Although this method produces conditions under which cyclic photophosphorylation is observed, the purging operation and preillumination conditions required to elicit activity may lead to unwanted side effects, such as photoinactivation and uncoupling of the chloroplasts. Prolonged purging of chloroplasts with inert gas can be avoided by the use of the enzymatic oxygen trapping system (glucose, glucose oxidase, catalase, and ethanol) described by Kok et al. (1965). Generation of reducing power for the cycle remains a problem, however. Chemical reductants of ferredoxin and low-potential quinones, such as dithionite or dithiothreitol, may produce undesirable side effects, and the possible deliterious effects of photosynthetic catalyst reduction have already been noted.

In the course of experiments to determine whether AQS $\left(E_{0}^{\prime}=-225 \mathrm{mV}\right)$ could be used as an efficient catalyst of PS I cyclic photophosphorylation, we noted an anomalous response of the anaerobic reaction system to light quality. White light produced ATP synthesis; red light did not. This observation led us to the discovery that AQS could be reduced 
photochemically in the absence of chloroplasts, a fact that has bien known for some time among research workers in the textile industry (Phillips et al., 1969). This finding has enabled us to devise a simple method, reported in this communication, for the assay of certain cyclic photophosphorylation reactions. The method we have developed produces anaerobiosis and reduced catalysts, while avoiding entirely the gassing and preillumination of labile photosynthetic membranes. This in turn permits the assay of high rates of DBMIB-sensitive PS I cyclic photophosphorylation catalyzed by autoxidizeable quinones.

\section{MATERIALS AND METHODS}

Chloroplasts were isolated from market spinach and stored at $-70^{\circ} \mathrm{C}$ in $0.5 \mathrm{~m} /$ aliquots as described by Guikema and Yocum (1976). Photoreactions were carried out in a stirred, thermostatted $\left(25^{\circ} \mathrm{C}\right) 1.75 \mathrm{~m} /$ glass cuvette equipped with a Clark-type oxygen electrode obtained from Yellow Springs Instrument Co. The cuvette was stoppered by a ground-glass cylinder containing a narrow bore opening $(2 \mathrm{~mm})$ which provided access for additions to the cuvette under anaerobic conditions. The polarizing valtage for the electrode was supplied by a battery-powered circuit similar to that described by Estabrook (1967), and the signal from the electrode was fed to a 10 in. chart recorder. The electrode sensitivity was adjusted to display $240 \mu M$ oxygen as a full-scale deflection on the chart recorder.

The reaction mixture $(1.75 \mathrm{~m} /)$ for assay of photophosphorylation activity consisted of Tricine $(45 \mathrm{mM}, \mathrm{pH} 8)$. $\mathrm{NaCl}(45 \mathrm{mM}), \quad \mathrm{MgCl}_{2}(3 \mathrm{mM}), \quad \mathrm{ADP}(1 \mathrm{mM})$ and $\mathrm{NaH}_{2}{ }^{32} \mathrm{PO}_{4}\left(5 \mathrm{mM}, 10^{6} \mathrm{cpm} / \mathrm{m} /\right)$. Glucose $(6 \mathrm{mM})$. glucose oxidase $(30 \mu \mathrm{g})$, and catalase $(250 \mu \mathrm{g})$ were also present, along with DCMU $(3 \mu M)$, which was added as a $5 \mu /$ aliquot from a stock solution $(1 \mathrm{mM})$ in $95 \%$ ethanol. This addition also supplied the ethanol $(0.3 \% \mathrm{v} / \mathrm{v})$ for the oxygen trapping system. 9,10-anthraquinone-2-sulfonate was added from a stock solution $(5 \mathrm{mM})$ in water to the desired final concentration, the cuvette was stoppered, and enzymatic oxygen removal was monitored by the electrode.

After anaerobiosis was achieved, the cuvette was subjected to the illumination protocols described in Results. Chloroplasts $(10-15 \mu /$ of a $2 \mathrm{mg} \mathrm{Chl} / \mathrm{m} /$ suspension) were added to the cuvette through the opening in the groundglass stopper by means of a microliter syringe. Illumination was provided by an Oriel model 6235 light source. The intensity of the white light used in these experiments was $3 \mathrm{~kJ} / \mathrm{m}^{2} / \mathrm{s}$, as determined with a Yellow Springs Instrument Co. Model 65 radiometer. Red light $\left(1 \mathrm{~kJ} / \mathrm{m}^{2} / \mathrm{s}\right)$ was provided by placing a red gelatin filter with a cut-on at $600 \mathrm{~nm}$ between the light source and the reaction cuvette. In all experiments, the light was filtered through $7 \mathrm{~cm}$ of a $0.2 \%$ solution of $\mathrm{CuSO}_{4}$ to remove infrared radiation.

After illumination, reaction mixtures were quenched with $0.2 \mathrm{~m} /$ of a $30 \%$ solution of trichloroacetic acid, and unreacted phosphate was extracted from the acidified material by the procedure of Avron (1960). Aliquots of the extracted material were dried on planchettes, and the amount of ATP synthesized was determined by gas-flow counting.

Glucose oxidase (110 units $/ \mathrm{mg}$ ) was obtained from Worthington Biochemical Corp. Catalase (2500 units/mg), antimycin A, menadione bisulfite and ADP were from Sigma. 9,10-anthraquinone-2-sulfonate was obtained from Eastman Organics and DCMU was from $K$ and $K$ Laboratories. Dibromothymoquinone was a generous gift from Dr. N. E. Good. All other chemicals used in these studies were of the purest grades commercially available.

\section{RESULTS}

Table 1 presents the results of an experiment where oxidized AQS was added to an anaerobic cuvette along with DCMU-poisoned chloroplasts. Illumination of the reaction mixture with strong white light produced rates of ATP synthesis 3-fold higher than has been previously reported for AQS (Hauska et al., 1974). We could not readily explain this result, since the system under assay was electron deficient. When red light was substituted for white light, as shown in Table 1, no ATP synthesis was obtained, nor was any activity observed with either white or red light when AQS was omitted from the assay system. These findings suggest that either white light illumination was promoting an AQS-catalyzed reaction which produced non-extractable counts in our reaction mixture, or that white light was inducing a change in AQS which produced a catalyst of PS I-dependent cyclic photophosphorylation.

These hypotheses were tested as follows. When a solution of AQS in the phosphorylation reaction mixture was illuminated with white light under anaerobic conditions in the absence of chloroplasts, no incorporation of radioisotope into non-extractable material was observed (data not shown). If, however, AQS in phosphorylation reaction mixtures was subjected to illumination with either white or red light in the absence of chloroplasts, chloroplasts were then added to the reaction mixture, and a second period of illumination was given, the results shown in Table 2 were obtained. ATP synthesis was observed only when the AQS solution minus chloroplasts was illuminated with white light. The second illumination, of the complete reaction mixture containing chloroplasts, was insensitive to light quality since approximately equal rates of photophosphorylation were obtained with either white or red light.

The data in Tables 1 and 2 convincingly demonstrate that white light illumination of AQS in the absence of chloroplasts generates a catalyst of PS I cyclic photophosphorylation. This activation process does not require chloroplasts, as evidenced by both the higher rates of ATP synthesis obtained when AQS is illuminated in the absence of chloroplasts (Table 2 ), and by the failure of red light, which activates

Table 1. Effect of exciting light color on AQS-mediated PS I cyclic photophosphorylation

\begin{tabular}{lcc}
\hline $\begin{array}{c}\text { Exciting Light } \\
(1 \mathrm{~min} \text { illumination })\end{array}$ & Catalyst & $\begin{array}{c}\text { ATP Synthesis } \\
(\mu \mathrm{mol} / \mathrm{h} \cdot \mathrm{mg} \text { Chl })\end{array}$ \\
\hline White & $115 \mu M$ AQS & 74 \\
White & None & 0 \\
Red & $115 \mu M$ AQS & 0 \\
Red & None & 0 \\
\hline
\end{tabular}

Assays were carried out under the conditions specified in Materials and Methods. Light intensities were $3 \mathrm{~kJ} / \mathrm{m}^{2} / \mathrm{s}$ (white light) and $1 \mathrm{~kJ} / \mathrm{m}^{2} / \mathrm{s}$ (red light). The Chl concentration was $16 \mu \mathrm{g} / \mathrm{m} /$. 
Table 2. Effect of preillumination of AQS solutions on PS I cyclic photophosphorylation

\begin{tabular}{llc}
\hline $\begin{array}{c}\text { Preillumination condition } \\
\text { (without chloroplasts) }\end{array}$ & $\begin{array}{c}\text { Exciting light } \\
\text { (chloroplasts present) }\end{array}$ & $\begin{array}{c}\text { ATP Synthesis } \\
(\mu \mathrm{mol} / \mathrm{h} \cdot \mathrm{mg} \text { Ch) }\end{array}$ \\
\hline 2 min white light & 1 min white light & 191 \\
2 min white light & 1 min red light & 200 \\
2 min red light & 1 min red light & 6 \\
\hline
\end{tabular}

Assays were carried out as described in Materials and Methods. Light intensities and $\mathrm{Chl}$ concentrations are those given in Table 1 .

$\mathrm{Chl}$ photochemistry, to produce any ATP synthesis in the absence of a white light illumination step (Tables 1 and 2).

Attempts were next made to determine the optimal concentration of AQS which would support ATP synthesis in our assay system. The white light illumination time was fixed at $2 \mathrm{~min}$, varying concentrations of ARS were subjected to this period of illumination, and chloroplasts were then added to the cuvette. A second, $1 \mathrm{~min}$ period of red light illumination was used to catalyze phosphorylating cyclic electron transport. The results of these experiments are shown in Fig. 1. Under our conditions for assay, optimal rates of ATP synthesis are obtained at an AQS concentration of approximately $115 \mu M$. At higher concentrations of catalyst, some decrease in activity is seen (Fig. 1).

We then examined the effect of white light illumination time on photophosphorylation activity under conditions where the AQS concentration was held constant at $115 \mu \mathrm{M}$. The results of these studies, presented in Fig. 2, show that a 2 min white light illumination period is adequate to produce maximal rates of ATP synthesis under the conditions described

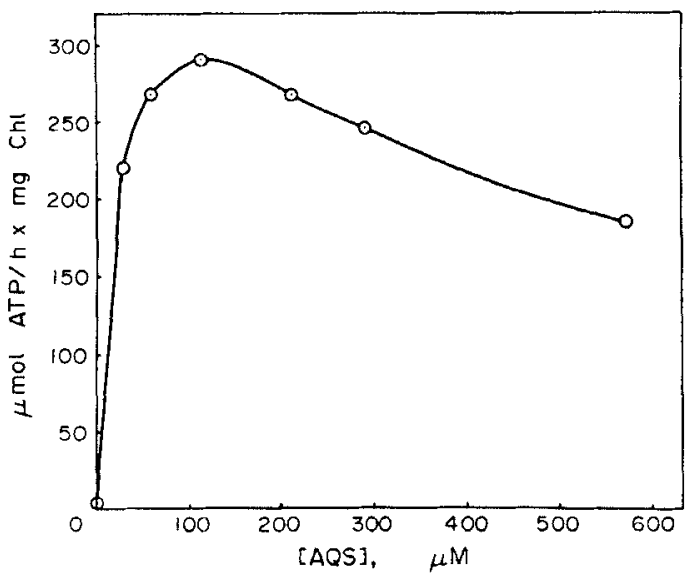

Figure 1. Effect of AQS concentration on the rate of PS I cyclic photophosphorylation. The reaction mixture is described in Materials and Methods. All reaction mixtures were subjected to a 2 min preillumination period in the absence of chloroplasts (white light) and to a $1 \mathrm{~min}$ period of illumination with red light in the presence of chloroplasts. Light intensities and $\mathrm{Chl}$ concentration are given in Table 1. here. We have varied both white light illumination time and AQS concentration over wide ranges, and have invariably found that a $2 \mathrm{~min}$ illumination period with white light at AQS concentrations from 100 to $120 \mu M$ produces optimal rates of cyclic photophosphorylation activity $(240-300 \mu \mathrm{mol} / \mathrm{h} \cdot \mathrm{mg} \mathrm{Chl})$.

Table 3 presents the results of experiments to determine the effect of the electron transport inhibitors antimycin A and DBMIB on PS I cyclic photophosphorylation catalyzed by AQS under our conditions of assay. The reaction is only sparingly sensitive to antimycin $A$ at concentrations which strongly inhibit ferredoxin-catalyzed photophosphorylation (Böhme et al., 1971), but DBMIB exerts a strong inhibitory effect on the reaction. This finding is in agreement with the results of other studies (Hauska et al., 1974; Voegeli et al., 1977) in which menadione and anthraquinone were employed as catalysts of cyclic electron transport.

A search of the research literature conducted in the course of our studies revealed that other investigators have observed the photoreduction of AQS in a system where alcohols served as electron donors (Phillips $e t$ al., 1969). Since our reaction mixtures contained ethanol, we carried out a series of experiments to determine whether ethanol or other components of our reaction mixture served to donate electrons for the

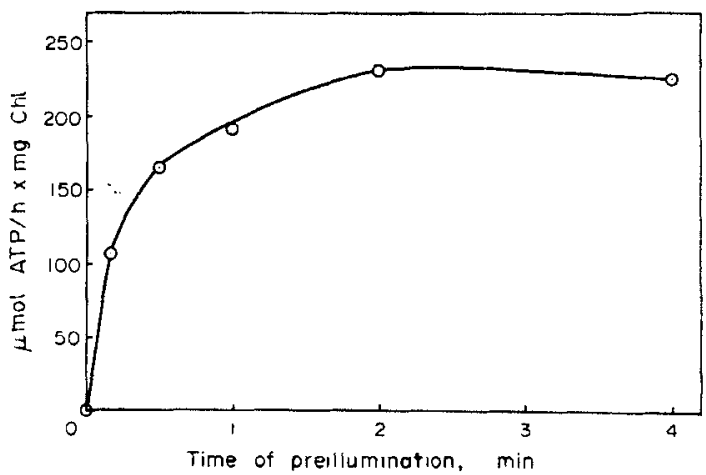

Figure 2. Effect of white light preillumination time on the rate of PS I cyclic photophosphorylation catalyzed by $115 \mu \mathrm{M}$ AQS. The reaction mixture is described in Materials and Methods. Chloroplasts were added after the illumination times shown and a $1 \mathrm{~min}$ period of red light illumination was used to catalyze phosphorylating electron transport. Light intensities and $\mathrm{Chl}$ concentration are given in Table 1. 
Table 3. Effect of antimycin A and DBMIB on AQS-catalyzed PS I cyclic photophosphorylation

\begin{tabular}{lcc}
\hline \multicolumn{1}{c}{ Addition } & $\begin{array}{c}\text { ATP Synthesis } \\
(\mu \mathrm{mol} / \mathrm{h} \cdot \mathrm{mg} \mathrm{Chl})\end{array}$ & $\%$ Control \\
\hline None & 245 & 100 \\
$6 \mu M$ Antimycin A & 168 & 69 \\
14 $\mu$ M Antimycin A & 156 & 64 \\
$1.7 \mu M$ DBMIB & 26 & 11 \\
$6 \mu M$ DBMIB & 4 & 2 \\
\hline
\end{tabular}

Assays were carried out under the conditions described in Materials and Methods. Reaction mixtures were subjected to a 2 min preillumination with white light in the absence of chloroplasts, and to a 1 min period of illumination with red light with chloroplasts present. Light intensities and the $\mathrm{Chl}$ concentration are given in Table 1.

photoreduction of AQS. For these studies, the photoactivity of AQS was monitored by observing the oxygen uptake which resulted from reoxidation of photoreduced AQS, a reaction which has been shown to produce $\mathrm{H}_{2} \mathrm{O}_{2}$ (Phillips et al, 1969). The results of this experiment, shown in Table 4 , indicate that of the compounds in our reaction mixture tested for activity, the buffer Tricine supports the highest rates of oxygen uptake. Neither ethanol nor inorganic phosphate produced appreciable rates of oxygen uptake, and we interpret the results in Table 4 to indicate that Tricine is the primary electron donor for photoreduction of AQS in our reaction system, a result which agrees with the earlier findings of Swader and Kumamoto (1975).

In order to confirm that illumination of AQS in the presence of Tricine was in fact producing the anthrahydroquinone species, a series of experiments was done to examine and compare the spectra of AQS obtained before and after exposure to either white light or a chemical reductant $\left(\mathrm{NaBH}_{4}\right)$. These investigations were carried out in $4 \mathrm{~m} /$ Thunberg cuvettes containing $3 \mathrm{~m} /$ of a $125 \mu \mathrm{M}$ solution of AQS in $50 \mathrm{mM}$ Tricine buffer. The cuvettes were made anaerobic by repeated evacuation and flushing with helium, after which the AQS solution was subjected either to chemical reduction (by addition of a few grains of $\mathrm{NaBH}_{4}$ from the sidearm of the cuvette), or to a period of illumination with white light. Representative spectra from these experiments, obtained with a Cary 17 ratio recording spectrophotometer, are shown in Fig. 3. Curve $A$ is the spectrum of untreated, oxidized AQS. Curve $B$ is the spectrum of AQS reduced by addition of $\mathrm{NaBH}_{4}$, and curve $\mathrm{C}$ is the spectrum produced by a $5 \mathrm{~min}$ period of illumination with white light. Spectra $B$ and $C$ both reverted to spectrum $A$ ( $>90 \%$ reoxidation) when air was admitted to the cuvette, indicating that in both chemically reduced and photoreduced solutions, the anthrahydroquinone could be reoxidized. It is clear from these results that illumination of AQS in the presence of Tricine serves to produce a reduced species whose spectrum resembles that of chemically reduced AQS (Fig. 3).

The relative ease and simplicity of our method for assay of AQS-supported PS I cyclic photophosphorylation reactions made it possible for us to quickly assess the applicability of this method to other quinones. In the presence of Tricine buffer, we observed spectrophotometrically the formation of the reduced forms of menadione bisulfite, 1,4-naphthoquinone-2-sulfonate, and 1,2-naphthoquinone-4-sulfonate upon illumination with white light (data not shown). When we tested these compounds for photophosphorylation activity under the conditions developed for assay of AQS-catalyzed ATP synthesis, the results shown in Table 5 were obtained. Only menadione bisulfite is catalytically active in our assay system, producing rates of ATP synthesis equivalent to those obtained with AQS.

\section{DISCUSSION}

The method for photoreduction of catalysts (AQS and menadione bisulfite) of PS I cyclic photophosphorylation which we describe here has three distinct advantages over previous techniques. As we have developed the procedure, chloroplasts are not subjected to purging with inert gases to achieve anaerobiosis nor are they preilluminated to generate the reduced form of the catalyst of cyclic electron transport. In addition, the extent of catalyst reduction can be conveniently controlled by varying the time of exposure of the reaction mixture to white light. Perhaps the best measure of the efficacy of our method is the success we have obtained in demonstrating high rates of PS I cyclic photophosphorylation supported by the autooxidizable catalyst AQS. Previous studies with this compound produced rates of ATP synthesis of about $25 \mu \mathrm{mol} / \mathrm{h} \cdot \mathrm{mg} \mathrm{Chl}$ (Hauska et al., 1974). We

Table 4. Oxygen uptake by a solution of AQS illuminated with white light

\begin{tabular}{lc}
\hline \multicolumn{1}{c}{$\begin{array}{c}\text { Addition to } 100 \mu M \\
\text { AQS Solution }\end{array}$} & $\begin{array}{c}\text { Rate of Oxygen Uptake } \\
(\mu \mathrm{mol} / \mathrm{min} \cdot \mu \mathrm{mol} \text { AQS })\end{array}$ \\
\hline None & 0.023 \\
$50 \mathrm{~m} M$ Ethanol & 0.048 \\
$12 \mathrm{~m} M$ Tricine $(\mathrm{pH} 8)$ & 0.570 \\
$12 \mathrm{~m} M$ Tricine $(\mathrm{pH} 8)+50 \mathrm{mM}$ Ethanol & 0.570 \\
$12 \mathrm{~m} M$ Phosphate $(\mathrm{pH} 8)$ & 0.027 \\
\hline
\end{tabular}

The reaction mixture $(1.75 \mathrm{~m} /)$ contained $\mathrm{AQS}$ and the additions shown in the Table. The light intensity was $3 \mathrm{~kJ} / \mathrm{m}^{2} / \mathrm{s}$. 


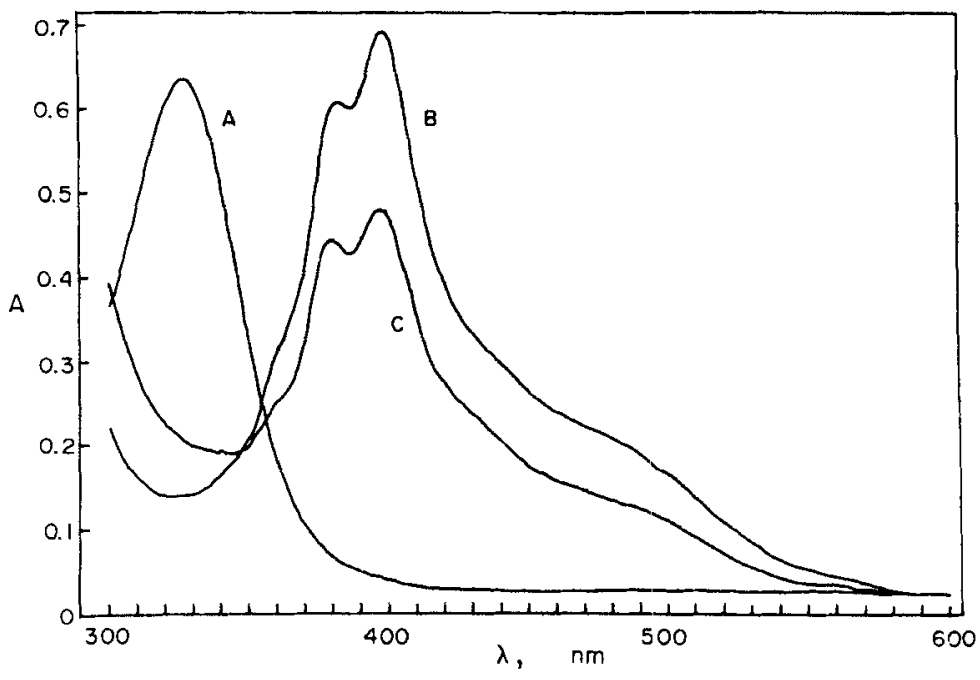

Figure 3. Spectra of oxidized (A), chemically reduced (B) and illuminated (C) solutions of $125 \mu M$ AQS in Tricine buffer. See text for conditions.

have obtained rates some 10 -fold higher using the photochemical method for reducing AQS, and our findings demonstrate that $\mathrm{AQS}$ is an efficient catalyst of photosystem I cyclic photophosphorylation in spite of the presence of a sulfonate group on this catalyst molecule, which should impair its lipid solubility.

Quinones other than AQS are amenable to photoreduction. Menadione bisulfite, 1,4-naphthoquinone2-sulfonate and 1,2-naphthoquinone-4-sulfonate are all photoreduced in the presence of Tricine buffer, as evidenced by the absorption spectra obtained following white light illumination (data not shown). Of these compounds, however, only menadione bisulfite is an effective catalyst of PS I cyclic photophosphorylation (Table 5). This finding confirms the earlier results of Hauska et al. (1974). These investigators suggested that the failure of higher potential quinones to support ATP synthesis was due to the inability of these compounds to donate electrons to endogenous constituents of the photosynthetic electron transport chain. Our results are consistent with this hypothesis.

It is clear from our results that the presence of a sulfonate or bisulfite group on the catalyst molecule does not interfere with the ability of either anthraquinone or menadione to stimulate PS I cyclic elec- tron transport. It appears likely that the catalysts of activity utilized for the present studies are dependent upon a portion of the photosynthetic electron transport chain that contains component(s) which are sensitive to DBMIB; a similar result has been reported for PS I cyclic activity catalyzed by menadione (Böhme et al., 1971; Voegeli et al., 1977). It is also apparent that the electron transport pathway utilized by AQS and menadione bisulfite differs somewhat from the pathway which is operative when ferredoxin is a catalyst of cyclic photophosphorylation. The ferredoxin-dependent reaction has been shown to be highly sensitive to antimycin A (Böhme et al, 1971). Although AQS $\left(E_{0}^{\prime}=-225 \mathrm{mV}\right)$ is certainly capable of reducing cytochrome $b_{6}$ (Knaff, 1972), one of the components thought to participate in the ferredoxincatalyzed cycle, our results indicate that AQS-catalyzed activity is minimally sensitive to antimycin $A$ (Table 3).

We cannot determine from our data the mechanism of AQS photoreduction in the presence of Tricine buffer. Wilkinson (1962) suggested that in the presence of alcohols as electron donors, AQS photoreduction might proceed as shown in Eq. 1

$$
2 \mathrm{AQS} \stackrel{\text { light }}{\longrightarrow} 2 \mathrm{AQSH} \cdot \longrightarrow \mathrm{AQSH}_{2}+\mathrm{AQS}
$$

Table 5. Catalysis of PS I cyclic photophosphorylation by quinones

\begin{tabular}{lcccc}
\hline \multicolumn{1}{c}{$\begin{array}{c}\text { Catalyst added } \\
(100 \mu M)\end{array}$} & $\begin{array}{c}E_{0}^{\prime} \\
(\mathrm{mV})\end{array}$ & $\begin{array}{c}\text { ATP synthesis } \\
(\mu \mathrm{mol} / \mathrm{h} \cdot \mathrm{mg} \text { Chl })\end{array}$ & $\begin{array}{c}\text { 1.7 } \mu M \text { DBMInhibition by: } \\
\text { 6 } \mu M \text { Antimycin A }\end{array}$ \\
\hline 9,10-Anthraquinone-2-Sulfonate & -225 & 311 & 89 & 31 \\
Menadione Bisulfite & +30 & 320 & 86 & 35 \\
1,4-Naphthoquinone-2-Sulfonate & +113 & 0 & - & - \\
1,2-Naphthoquinone-4-Sulfonate & +208 & 5 & - & - \\
\hline
\end{tabular}

Assays were carried out in the reaction mixture described in Materials and Methods. All catalysts were subjected to a $2 \mathrm{~min}$ preillumination period in the absence of chloroplasts, and a $1 \mathrm{~min}$ period of illumination with red reaction light in the presence of chloroplasts. The Chl concentration was $18 \mu \mathrm{g} / \mathrm{m} /$. 
It is likely that this reaction, or one very similar to it, is responsible for the results we have obtained in the presence of Tricine buffer. The ability of Tricine to serve as an electron donor for photochemical reduction reactions is not surprising. Nelson et al. (1972) and Yamazaki and Tolbert (1970) have shown that this buffer could serve as an efficient electron donor in a system for the photoreduction of flavin mononucleotide. We have tested other buffers for electron donor activity by monitoring oxygen uptake due to the reoxidation of photoreduced AQS. Both 2-( $N$-morpholino)ethanesulfonic acid (MES) and $N$-2-hydroxyethylpiperazine- $N$ '-ethanesulfonic acid (HEPES) produced rates of oxygen uptake in their respective buffering ranges which were equal to, or greater than, the rates observed with Tricine at $\mathrm{pH}$ 8. It is therefore likely that the photochemical method for AQS reduction can be applied to assay systems over the entire $\mathrm{pH}$ range of interest for PS I cyclic photophosphorylation reactions.

\section{REFERENCES}

Avron, M. (1960) Biochim. Biophys. Acta 40, 257-272.

Böhme, H., S. Reimer and A. Trebst (1971) Z. Naturforsch. 26b, 341-352.

Estabrook, R. (1967) In Methods in Enzymology (Edited by R. W. Estabrook and M. E. Pullman), Vol. X. pp. 41-47. Academic Press, New York.

Guikema. J. A. and C. F. Yocum (1976) Biochemistry 15, 362-367.

Hauska. G. A., S. Reimer and A. Trebst (1974) Biochim. Biophys. Acta 357, 1-13.

Knaff, D. (1972) FEBS Lett. 23, 92-94.

Kok, B., H. J. Rurainski and O. Owens (1965) Biochim. Biophys. Acta 109, 347-356.

Nelson. N., Z. Drechsler and J. Neumann (1970) J. Biol. Chem. 245, 143-151.

Nelson. N., H. Nelson and E. Racker (1972) Photochem. Photobiol. 16, 481-489.

Ouitrakul. R. and S. Izawa (1973) Biochim. Biophys. Acta 305, 105-118.

Phillips, G. O., N. W. Worthington, J. K. McKellar and R. R. Sharpe (1969) J. Chem. Soc. (A), $767-773$.

Swader. J. A. and J. Kumamoto (1975) Photochem. Photobiol. 21, 313-315.

Tagawa, K., H. Y. Tsujimoto and D. I. Arnon (1963) Proc. Natl. Acad. Sci. U.S. 50, 544-549.

Voegeli, K. K., D. O'Keefe, J. Whitmarsh and R. A. Dilley (1977) Arch. Biochem. Biophys. 183, 333-339.

Wilkinson. F. (1962) J. Phys. Chem. 66, 2569-2574.

Yamazaki, R. K. and N. E. Tolbert (1970) Biochim. Biophys. Acta 197, 90-92. 